\title{
Electrophysiological characterisation of central sensitisation in canine spontaneous osteoarthritis
}

James R. Hunt ${ }^{a, \star}$, Megan Goff ${ }^{a}$, Helen Jenkins ${ }^{a}$, John Harris ${ }^{b}$, Toby G. Knowles ${ }^{a}$, B. Duncan X. Lascelles ${ }^{\mathrm{c}, \mathrm{d}, \mathrm{e}, \mathrm{f}}$, Masataka Enomoto ${ }^{c}$, Michael Mendl ${ }^{a}$, Helen R. Whay ${ }^{a}$, Joanna C. Murrell ${ }^{a}$

\begin{abstract}
In man, central sensitisation (CS) contributes to the pain of osteoarthritis (OA). Dogs with spontaneous OA may also exhibit CS. Electrophysiological reflex measurements are more objective than behavioural assessments and can be used to evaluate CS in preclinical and clinical studies. It was hypothesised that dogs suffering from OA would exhibit electrophysiological characteristics indicative of CS, associated with reduced diffuse noxious inhibitory controls (DNICs). One hundred and seventeen client-owned dogs were recruited to the study. Hind limb nociceptive withdrawal reflex thresholds, stimulus response, and temporal summation characteristics were recorded, during alfaxalone anaesthesia, from 46 OA dogs, 29 OA dogs receiving nonsteroidal anti-inflammatory drugs (OANSAIDs), and 27 breed- and weight-matched control dogs. Efficacy of DNIC was evaluated in 12 control and 11 of the OA dogs, by application of a mechanical conditioning stimulus to the contralateral forelimb. Nociceptive withdrawal reflex thresholds were higher in OA compared with control dogs $(P=0.02)$. Stimulus response characteristics demonstrated an augmented response in OANSAID dogs compared with OA $(P<0.001)$ and control $(P<0.001)$ dogs. Temporal summation demonstrated exaggerated $\mathrm{C}$ fibre-mediated responses in both OA $(P<0.001)$ and OANSAID $(P=0.005)$ groups, compared with control animals. Conditioning stimulus application resulted in inhibition of test reflex responses in both $\mathrm{OA}$ and control animals $(P<0.001)$; control animals demonstrated greater inhibition compared with OA $(P=0.0499)$. These data provide evidence of neurophysiological changes consistent with CS in dogs with spontaneous OA and demonstrate that canine OA is associated with reduced DNIC.
\end{abstract}

Keywords: Central sensitisation, Diffuse noxious inhibitory controls, Dog, Nociceptive withdrawal reflex, Osteoarthritis

\section{Introduction}

Spontaneous canine osteoarthritis (OA) has been proposed as a model of human $\mathrm{OA}^{38}$ In man, in addition to mechanisms local to affected joints, central sensitisation (CS) may exacerbate pain. ${ }^{29}$ Some dogs affected by OA respond to centrally acting antihyperalgesic drugs ${ }^{26}$ and have altered nociceptive thresholds, ${ }^{21}$ suggesting CS; however, there is no "gold standard"

Sponsorships or competing interests that may be relevant to content are disclosed at the end of this article.

a Bristol Veterinary School, University of Bristol, Bristol, United Kingdom, ${ }^{b}$ Arthritis Research UK Pain Centre, and Division of Animal Sciences, School of Biosciences, University of Nottingham, Loughborough, United Kingdom, ${ }^{C}$ Comparative Pain Research and Education Centre, College of Veterinary Medicine, North Carolina State University, Raleigh, NC, United States, ${ }^{d}$ Comparative Medicine Institute, North Carolina State University, Raleigh, NC, United States, ${ }^{e}$ Center for Pain Research and Innovation, UNC School of Dentistry, Chapel Hill, NC, United States, ${ }^{f}$ Center for Translational Pain Research, Department of Anesthesiology, Duke University, Durham, NC, United States

*Corresponding author. Address: University of Bristol, Langford House, Langford, Bristol BS40 5DU, United Kingdom. Tel.: +44 (0)117928 9673. E-mail address: Ivjrh@bristol.ac.uk (J.R. Hunt).

Supplemental digital content is available for this article. Direct URL citations appear in the printed text and are provided in the HTML and PDF versions of this article on the journal's Web site (Www.painjournalonline.com).

PAIN 159 (2018) 2318-2330

Copyright (@) 2018 The Author(s). Published by Wolters Kluwer Health, Inc. on behalf of the International Association for the Study of Pain. This is an open-access article distributed under the terms of the Creative Commons Attribution-Non Commercial License 4.0 (CCBY-NC), where it is permissible to download, share, remix, transform, and buildup the work provided it is properly cited. The work cannot be used commercially without permission from the journal.

http://dx.doi.org/10.1097/j.pain.0000000000001336 approach for identifying and quantifying CS in dogs. Therefore, it is currently unknown whether OA in dogs is also associated with CS, yet this information is essential if canine OA is to be used as a valid model of human $O A$.

The RIII withdrawal response threshold and magnitude, and temporal summation (TS) to repeated stimuli are altered in pain syndromes associated with CS in man and may be used as objective markers of $\mathrm{CS}^{37}$ In dogs, the nociceptive withdrawal reflex $(\mathrm{NWR})^{6}$ and $\mathrm{TS}$ of the $\mathrm{NWR}^{8}$ have been suggested as potential biomarkers for CS. We have previously developed methods to evaluate these measures during anaesthesia. ${ }^{19}$ There are, presently, no reports of alterations in NWR or TS associated with painful disease in dogs, and the potential for the technique to characterise the state of spinal excitability remains untested.

Diffuse noxious inhibitory controls (DNIC) represent an endogenous supraspinal antinociceptive mechanism activated by heterotopic noxious ("conditioning") stimulation. ${ }^{5,15}$ Efficacy of conditioned pain modulation (CPM) in man (considered the equivalent of $\mathrm{DNIC}$ ) is a predictor of acute ${ }^{14}$ and chronic postoperative ${ }^{41}$ pain, and is commonly reduced in chronic pain states, including OA. ${ }^{2}$ There are no investigations of DNIC efficacy associated with OA in dogs. Conditioned pain modulation may be modulated by cognitive influences, ${ }^{30}$ which are challenging to control for experimentally. Therefore, it is desirable to develop a non-tissue-damaging paradigm, which may be applied to anaesthetised animals.

The primary aim of the studies described here was to compare electrophysiological responses, including TS of C-fibre responses, in a cohort of client-owned pet dogs suffering from spontaneous OA, with a matched group of control pet dogs. Dogs within the OA cohort were divided into those receiving daily nonsteroidal 
anti-inflammatory drugs (NSAIDs) to manage OA-associated pain (OA dogs receiving nonsteroidal anti-inflammatory drugs [OANSAIDs]) and dogs not receiving drug treatment (OA). We hypothesised that dogs with OA would exhibit electrophysiological characteristics indicative of CS, and that these characteristics would be exaggerated in the OANSAID group compared with the OA group because of the greater pain that was likely experienced by OANSAID dogs, despite ongoing NSAID administration.

Our second aim was to develop an effective protocol to evaluate DNIC in dogs. Conditioned pain modulation has been elicited by mechanical conditioning stimulation (MCS) ${ }^{33}$; therefore, we sought to investigate whether MCS would evoke DNIC, and whether DNIC efficacy was decreased by OA. We hypothesised that in control dogs, application of MCS would inhibit the NWR, and that the degree of inhibition would be reduced in dogs affected by $\mathrm{OA}$.

\section{Methods}

\subsection{Nociceptive withdrawal reflex/temporal summation investigation}

\subsubsection{Ethics}

The study was conducted under the terms of the Animal (Scientific Procedures) Act, 1986 (as amended, 2013) (A(SP)A) licence number PPL 30/3157, and the experimental protocol was approved by the University of Bristol Animal Welfare and Ethical Review Body.

\subsubsection{Recruitment criteria}

Advertisements to recruit participants for the study were posted on social media (Facebook and Twitter), within the local University of Bristol intranet, and within local veterinary practices. For the OA group, suitable dogs were $12 \mathrm{~kg}$ body weight and above, of any age, body condition and sex exhibiting suspected painful unilateral or bilateral coxofemoral or stifle degenerative joint disease as evidenced by lameness/stiffness/difficulty in rising or ascending steps. Dogs with primarily forelimb lameness were excluded. During the study recruitment phase, a large proportion of dogs screened were already receiving daily treatment with NSAIDs for musculoskeletal pain, and the decision was made to recruit these animals and permit them to continue daily NSAID treatment, but to designate them as a separate group (OANSAID) for analysis within the study. This decision was based on the fact that pain and disability were still present in these individuals, despite treatment with the NSAID.

The inclusion criteria for the control group were based on the demographics of a cohort of $O A$ dogs recruited to a separate study at the University of Bristol, ${ }^{16}$ where a mean (SD) age of $9.5 \pm 3$ years and weight of $27.5 \pm 11.6 \mathrm{~kg}$ were recorded. For this study, dogs were recruited to the control group that were 6 years old or

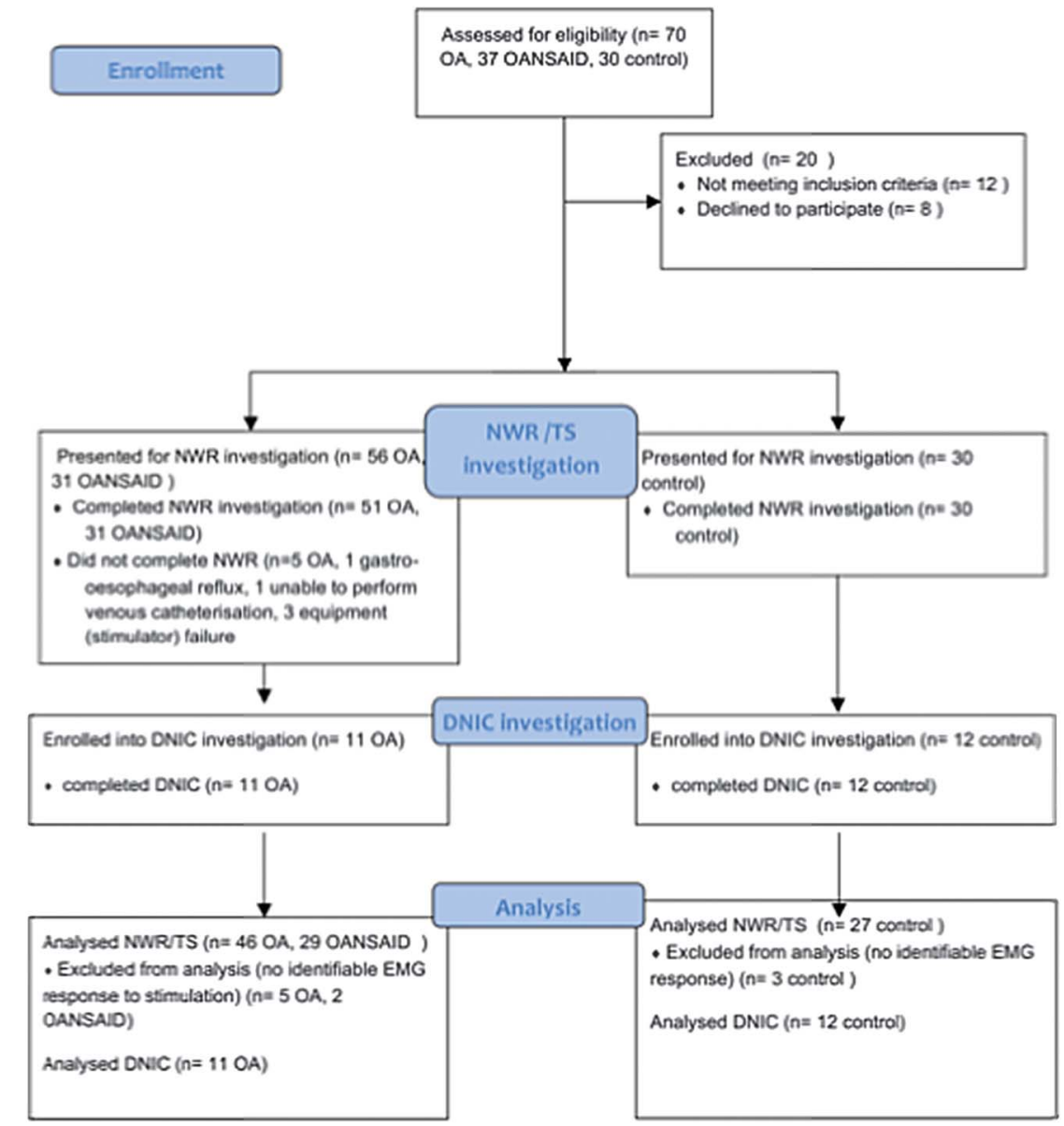

Figure 1. Illustration of the number of animals recruited to each OA category and attrition through different stages of the study. DNIC, diffuse noxious inhibitory control; EMG, electromyography; NWR, nociceptive withdrawal reflex; OA, osteoarthritis; OANSAID, OA dogs receiving nonsteroidal anti-inflammatory drug; TS, temporal summation. 
greater and $12 \mathrm{~kg}$ body weight and above, exhibiting no evidence of lameness or stiffness and with no other painful condition (eg, otitis externa) and no previous diagnosis of OA. Figure 1 illustrates the outcomes for all dogs that attended screening and the subsequent numbers that were used at each stage of the study.

\subsubsection{Study protocol}

Owners of eligible dogs were asked to attend a screening appointment, at which the purpose and procedures of the study were explained verbally and in writing, and signed consent to participate was obtained before any study procedures being performed. Dogs underwent physical and musculoskeletal examination by a veterinarian (J.R.H.). Body condition score (1, emaciated-9, morbidly obese ${ }^{24}$ ) was assessed by manual palpation. Any dogs with identifiable comorbidities that would have increased risks associated with general anaesthesia, or dogs with neurological dysfunction evidenced by weak or absent conscious proprioception, were excluded from the study. Microchip details were confirmed as a means of permanently identifying participating dogs to comply with the terms of the A (SP)A. Owners were asked to complete the ACVS Canine Orthopaedic Index, ${ }^{9}$ the Helsinki Chronic Pain Index, ${ }^{17}$ the Liverpool Osteoarthritis in Dogs (LOAD) questionnaire, the Canine Brief Pain Inventory (CBPI), ${ }^{10}$ and the sleep and night time restlessness evaluation (SNoRE). ${ }^{22}$ Jugular blood samples were obtained and submitted for routine biochemistry and haematology before scheduling general anaesthesia.

\subsubsection{Musculoskeletal examination}

Scores for lameness (0-10) and mobility (0-3) were assigned by a veterinarian (J.R.H.), according to the criteria shown in Appendix 1 (available online as supplemental digital content at http://links.Iww.com/PAIN/A622).

Examination of each joint was performed, and individual appendicular joints were scored from 0 (not affected) to 3 (severely affected) for the criteria "range of motion," "pain on extension or flexion," "crepitus," "effusion," and "thickening." The sum of the joint disease scores produced an overall OA score between 0 and 192, whereas the sum of the pain scores for each joint produced an overall joint pain score between 0 and 48 .

\subsubsection{General anaesthesia}

Seven days after the initial screening appointment, dogs were admitted to the Wellcome Comparative Anaesthesia Research Laboratory, Langford, Bristol, to undergo radiography and NWR testing under general anaesthesia.

On admission, confirmation that dogs had had food withheld for 8 hours was sought from owners and a veterinary examination was repeated.

Acepromazine (ACP 2 mg/mL solution; Elanco Animal Health, Basingstoke, United Kingdom) was administered intramuscularly (0.03 $\mathrm{mg} / \mathrm{kg})$, and dogs were left undisturbed for 30 minutes, following which a cephalic venous catheter was placed. Insufficient sedation to permit intravenous catheterisation warranted exclusion from the study.

Alfaxalone (Alfaxan; Jurox [UK] Ltd, Crawley, United Kingdom) (1-2 $\mathrm{mg} / \mathrm{kg}$ ) was administered intravenously over a period of 60 seconds until orotracheal intubation was possible. Oxygen was delivered through a circle breathing system and anaesthesia maintained with a constant rate infusion of alfaxalone $(0.1 \mathrm{mg} / \mathrm{kg} / \mathrm{min})$ during radiography, reducing to $0.09 \mathrm{mg} / \mathrm{kg} / \mathrm{min}$ for NWR testing. Body temperature was monitored every 30 minutes and supported with insulated electric blankets. After NWR testing, alfaxalone infusion was discontinued and the dogs constantly monitored until they were discharged to the owner once able to walk and having eaten. All dogs not ordinarily receiving NSAIDs were treated with meloxicam (Metacam $5 \mathrm{mg} / \mathrm{mL}$ solution; Boehringer Ingelheim, Bracknell, United Kingdom) $(0.2 \mathrm{mg} / \mathrm{kg})$ to treat any pain caused by positioning for radiography or NWR recording.

\subsubsection{Radiography}

Lateral and cranial-caudal views of the elbows and stifles; lateral views of the lumbosacral junction; and ventrodorsal views of the pelvis and coxofemoral joints were obtained in the Bristol Veterinary School imaging suite. Each of these 7 joints was assessed for severity of radiographic OA by 2 investigators (M.E. and B.D.X.L.) who were unaware of the OA group classification of the dogs. The investigators assigned scores from 0 (no radiographic signs of OA) to 10 (severe radiographic OA) for each joint, and thus, a global score for each dog out of 70 was recorded. The investigators performing NWR testing remained unaware of the results of the radiographs.

\subsubsection{Nociceptive withdrawal reflex testing}

Dogs were positioned in left lateral recumbency with the right pelvic limb resting on a sandbag, perpendicular to the table top. Paired stimulating electrodes (disposable subdermal needle electrode $12 \times 0.40$ mm; Natus Neurology Inc, Middleton, WI) were placed $10 \mathrm{~mm}$ apart subdermally into the plantar aspect of digit 3 of the right hind limb; paired recording electrodes were placed $20 \mathrm{~mm}$ apart into the body of the right cranial tibial (CT) muscle; and a ground electrode placed subcutaneously dorsal to the dorsal spinous process of L6. As previously described, ${ }^{23}$ the recorded signal was processed through a differential amplifier (DAM50; World Precision Instruments, Herts, United Kingdom), which applied a band-pass filter from 10 to $1 \mathrm{kHz}$ and gain of 1000, and was subsequently captured in Labchart 8 software (AD Instruments, Oxford, United Kingdom) following conversion by an analogue to digital converter with a sampling frequency of $1 \mathrm{kHz}$ (Powerlab 4/35; AD instruments).

\subsubsection{Electromyographic threshold}

Electrical stimuli were delivered through the toe electrodes using a constant current stimulator from an isolated $100 \mathrm{~V}$ source (Stimulus isolator FE180; AD instruments).

The threshold current at which a single 1-ms square wave stimulating pulse would evoke a visually discernable CT electromyographic (EMG) response (a response greater than the baseline amplitude) was identified by increasing current stepwise from 0 to a maximum of $10 \mathrm{~mA}$ in $0.5-\mathrm{mA}$ increments. After a response, the current was decreased by $0.1 \mathrm{~mA}$ increments until the response was no longer elicited. This up and down adjustment was continued until 3 stable readings for threshold were obtained at 60-second intervals.

\subsubsection{Stimulus response curve}

One stimulus event comprised five 1-ms stimuli (Train-of-5 [To5] ${ }^{23}$ ), which were delivered at a frequency of $100 \mathrm{~Hz}$. An EMG stimulus response determination was performed by triggering To5 events at 60-second intervals using currents of 0.1 (baseline), 1, 2, 3, 4, 5, 6, $7,8,9$, and $10 \mathrm{~mA}$. The complete series of stimulating currents was 
applied in the same ascending order on a second occasion after a 5-minute interval.

\subsubsection{Temporal summation}

A stimulus sequence of $8 \times 1 \mathrm{~ms} 10-\mathrm{mA}$ stimuli delivered at a rate of $1 \mathrm{~Hz}$ was repeated 3 times at 5 -minute intervals.

\subsubsection{Electromyographic analysis}

After recording, a 10- $\mathrm{Hz}$ high-pass digital filter was applied to the EMG traces, to further decrease movement artefact. The primary outcome measure for the study was the integral of the rectified EMG response that was extracted for each stimulus within each predefined time window. The EMG response was designated as early (representing an A-fibre response 0-100 ms) or late (C fibre; 100-500 ms) latency, time locked to the start of the stimulus train. ${ }^{23}$ Although the late response may also contain components of supraspinal origin, this differentiation was based on previous work in dogs $^{8}$ where conduction velocity of the different nerve fiber types and the length of the afferent distance were used to calculate latency ranges for the different ( $A$ and $C$ fiber) responses. Baseline activity in the absence of any electrically evoked response (the 0.1-mA stimulus for the stimulus response experiments and from within a 2-second period before application of the first of the 8 stimuli for TS experiments) was subtracted from each measurement.

\subsubsection{Statistical methods}

Recordings of NWR data were visually examined by 2 investigators (J.R.H. and J.H.) and where no identifiable response could be appreciated to a stimulation protocol, the data for that protocol for the individual dog were excluded from the analysis. Sex distribution data were analysed using $\chi^{2}$ tests. Comparisons of mean or median measures at single time points (eg, body weight, lameness, and owner-completed metrology instruments) between the 3 groups were performed using 1-way analysis of variance or Kruskal-Wallis tests followed by the Tukey (or Dunn) post hoc testing if applicable. The hierarchical structure of the data comprising the stimulus response and TS data was accounted for by using multilevel modelling within the MLwiN statistics package.$^{34}$ In the case of the stimulus response data, no transformation of the outcome variable was necessary, as the residuals from the analyses showed appropriate normality and homoscedasticity. It was necessary to apply natural log transformation to the TS data to meet the assumptions of the statistical models. Data analysed using parametric tests are presented as mean (95\% confidence interval [CI]), and the results of nonparametric testing are presented as median (25\%-75\% interquartile range). The final multilevel general linear models took the form of equations that described the effect of the statistically significant predictor variables on the outcome measures. The parameter estimates from the analyses are presented below, and the models are represented as graphs.

\subsubsection{Power calculation}

A power calculation for the overarching project, based on preliminary data using von Frey mechanical threshold data, indicated a total of 68 dogs, evenly divided between OA and control groups, would be required for a power of $90 \%$, at an alpha of 0.05 to detect a difference between control and OA dogs. However, this calculation assumed uniformity within the OA group, whereas we suspected that the OA group would be heterogenous, based on data from human OA patients and laboratory animal models of OA. In humans, up to $70 \%$ of patients with OA have at least one somatosensory abnormality. ${ }^{40}$ Based on this, we estimated that recruiting $100 \mathrm{OA}$ dogs would give us an appropriate cohort of CS-negative dogs (ie, approximately the same number as control dogs) and a cohort of CS-positive dogs that may be as large as 70 .

\subsection{Diffuse noxious inhibitory control investigation}

\subsubsection{Animals}

After completion of the NWR/TS protocol described above, some dogs underwent DNIC investigations during the same anaesthetic period (Fig. 1).

\subsubsection{Diffuse noxious inhibitory control protocol}

Five minutes after the final TS experiment, the DNIC protocol began by recording EMG responses in the CT muscle to test stimuli delivered at twice the individually determined threshold current (2xThr) at a rate of $1 \mathrm{~Hz}$ for 100 seconds. This occasion was denoted as "pre-DNIC." An identical test stimulation protocol (2xThr, $1 \mathrm{~Hz}, 100$ seconds) was repeated on 3 more occasions at 5 -minute intervals; however, during occasions 2 ("DNIC 1") and 3 ("DNIC 2"), the effect on CT responses of an additional mechanical conditioning stimulus, comprising a bulldog clip applied for 20 seconds to the third digit of the contralateral forelimb, was assessed (Fig. 2). The fourth and final occasion ("post-DNIC") was a repeat of the pre-DNIC stimulating protocol, without the addition of a conditioning stimulus.

Measurement of the force delivered by the bulldog clip at a jaw separation of $11 \mathrm{~mm}$ (mean jaw opening measured during the application to the digit) was achieved using a Loadcell $50 \mathrm{~N}$ gauge (Mecmesin; Slinfold, West Sussex, United Kingdom). The force recorded by the gauge at 11-mm separation was $33.4 \mathrm{~N}$, but this was also found to be consistent over the range of jaw opening from 2 to $12 \mathrm{~mm}$. Examination of the site of application following the DNIC protocol, and 7 days later, demonstrated no evidence of immediate or delayed ongoing pain or tissue damage.

\subsubsection{Statistical methods}

Sex distribution data were assessed using the Fisher exact test. Comparisons of weight and owner-completed metrology instrument scores between the 2 groups were performed using the Student $t$ test or Mann-Whitney $U$ test. The hierarchical structure of the DNIC testing data was accounted for within the statistical analysis by using general linear modelling within a multilevel modelling framework using the MLwiN statistics package. ${ }^{41}$ Predictor variables were retained within the model based on a Wald test at $\alpha \leq 0.05$. It was necessary to apply a natural log transformation to the EMG magnitude data, to meet the assumptions of the tests regarding normality and homoscedasticity of residuals. The pre-DNIC occasion was denoted as the reference occasion for comparisons within the model. Data subject to parametric tests are presented as mean $(95 \% \mathrm{Cl})$ and results subject to nonparametric testing are presented as median (25\%-75\% interquartile range).

\subsubsection{Power calculation}

A power calculation was performed for the overarching project; however, the DNIC investigation was performed to develop an effective but non-tissue-damaging model for evaluating DNIC in 


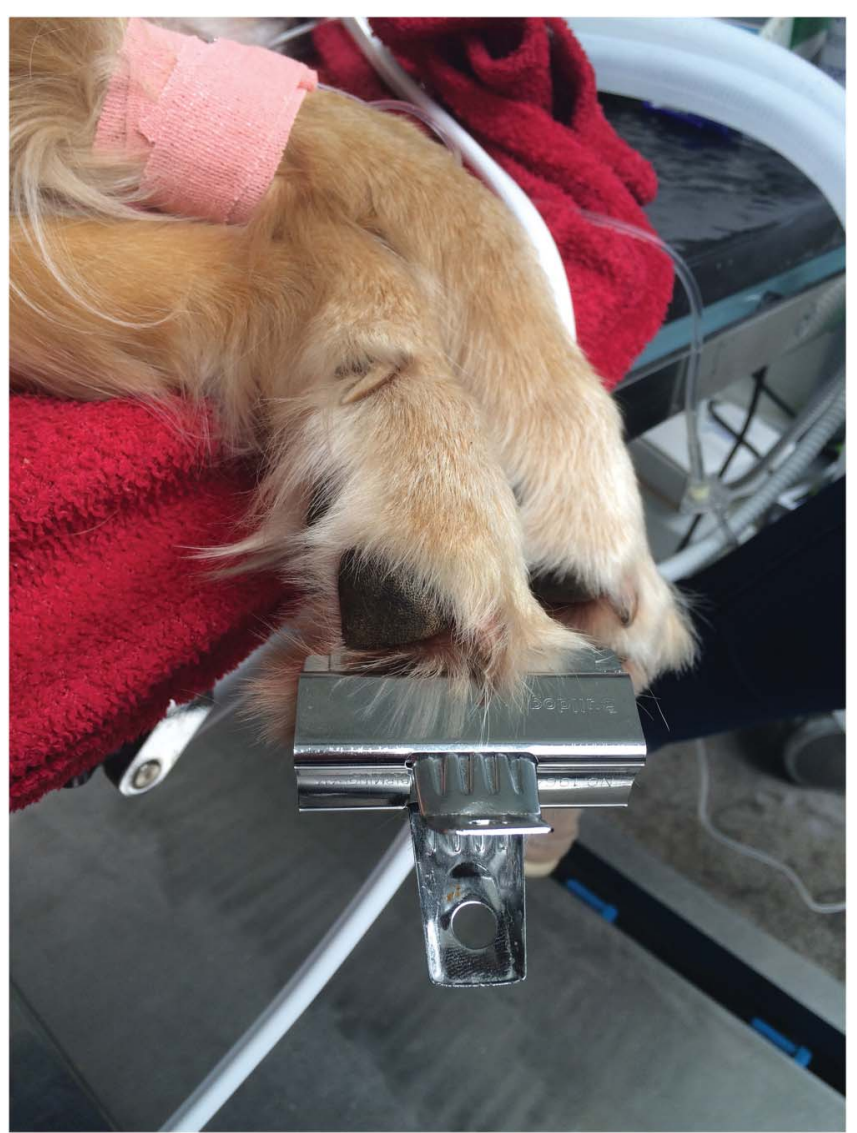

Figure 2. During anaesthesia, a bulldog clip conditioning stimulus was applied for 20 seconds to the third digit of the left cranial limb, whereas electrical test stimuli were delivered to the right pelvic limb.

dogs, and to provide pilot data for ongoing investigations, hence a power calculation was not performed specifically regarding the primary outcome measure (magnitude of EMG response) reported here.

\section{Results}

\subsection{Nociceptive withdrawal reflex/temporal summation investigation}

\subsubsection{Demographics}

Data were analysed from 27 control, 46 OA, and 29 OANSAID dogs. Breed and sex distribution are shown in Table 1. There was no significant difference in sex distribution, and breed distribution appeared to be visually well matched between groups. Weight and body condition scores were not different between groups (Table 1). Dogs in the control group were younger than dogs in both the OA and OANSAID groups (Table 1). The duration of NSAID treatment in the OANSAID group was variable between individuals, but animals had been receiving daily NSAIDs for at least 3 months before recruitment to the study.

\subsubsection{Veterinary assessment}

Degree of lameness, mobility score, total OA score, and total joint pain score were all significantly higher in OA and OANSAID groups compared with controls (Table 2); however, there were no differences between OA and OANSAID groups regarding these measures.
Table 1

\section{Demographic data.}

\begin{tabular}{lllll}
\hline & $\begin{array}{l}\text { Control } \\
\text { (n= 27) }\end{array}$ & OA (n= 46) & $\begin{array}{l}\text { OANSAID } \\
\text { (n= 29) }\end{array}$ & P \\
\hline Breed & & & & \\
Border collie & 7 & 10 & 5 & - \\
Labrador & 5 & 8 & 11 & - \\
Retriever & 3 & 3 & 1 & - \\
Lurcher & 3 & 2 & 0 & - \\
Spaniel & 1 & 5 & 3 & - \\
Other & 8 & 18 & 9 & - \\
\hline Sex & & & & \\
M & 3 & 3 & 3 & 0.61 \\
Mn & 7 & 18 & 14 & 0.61 \\
F & 1 & 3 & 2 & 0.61 \\
Fn & 16 & 22 & 10 & 0.61 \\
\hline Weight (kg) & $22.8(95 \% \mathrm{Cl}$ & $26.8(95 \% \mathrm{Cl}$ & $28.7(95 \% \mathrm{Cl}$ & 0.0563 \\
& $20.5-25.0)$ & $23.6-29.9)$ & $24.8-32.6)$ & \\
\hline Body condition & $5(4-6)$ & $5(5-6)$ & $5(4-6)$ & 0.19 \\
score (1-9) & & & & \\
\hline Age (y) & $7.8(95 \% \mathrm{Cl}$ & $9.8(95 \% \mathrm{Cl}$ & $9.6(95 \% \mathrm{Cl}$ & $<0.001^{\text {*** }}$ \\
\hline & $7.3-8.4)^{\mathrm{a}}$ & $9.2-10.3)^{\mathrm{b}}$ & $8.5-10.6)^{\mathrm{b}}$ & \\
\hline
\end{tabular}

Superscript letters indicate groupings within the data, shared superscripts indicate no significant difference between groups on post hoc testing, and differing superscripts indicate a difference with a $P$ value of less than 0.05 on post hoc testing. ${ }^{\star \star \star} P \leq 0.001$.

F, female; Fn, female neuter; M, male; Mn, male neuter; OA, osteoarthritis; OANSAID, OA dogs receiving nonsteroidal anti-inflammatory drug.

\subsubsection{Owner-completed clinical metrology instruments}

Questionnaire data were analysed by subsection if the questionnaire was constructed in a section format. Owner-attributed scores for all the questionnaire subsections were significantly higher (more dysfunction/pain) in OA and OANSAID animals compared with controls. In addition, the CBPI pain and ACVS function subsections were significantly higher in OANSAID compared with OA animals (Table 2), indicating that dogs receiving NSAID therapy experienced greater pain and greater dysfunction (eg, reduced mobility) than dogs with OA that were not receiving NSAID treatment.

\subsubsection{Radiographic scores}

Radiographic OA severity was significantly higher in both OA and OANSAID animals compared with controls but was not significantly different between OA and OANSAID animals (Table 2).

\subsubsection{Nociceptive withdrawal reflex recordings}

The early phase of the NWR could be reliably and repeatedly elicited in the CT muscle during the multiple trials at each stimulus intensity. Examples of raw traces obtained during NWR recording are provided (Figs. 3 and 4).

\subsubsection{Electrical threshold}

The threshold current to elicit an EMG response was significantly lower in control $(2.3[95 \% \mathrm{Cl} 1.8-2.9 \mathrm{~mA}])$ compared with $\mathrm{OA}$ dogs (3.8 [95\% Cl 3.0-4.6 mA] [ $\left.\left.F_{2,93}=3.859, P=0.02\right]\right)$, but neither group was different from OANSAID (3.2 [95\% Cl 2.4-3.9 $\mathrm{mA}]$ ) that had an intermediate value.

\subsubsection{Stimulus response}

Only the early component of the response was analysed, as the late response was absent in the majority of recordings (Table 3 ). 


\section{Table 2}

Musculoskeletal examination, owner-completed metrology instrument, and radiographic severity data.

\begin{tabular}{|c|c|c|c|c|}
\hline & Control & $\mathbf{O A}$ & OANSAID & $P$ \\
\hline Lameness $(0-10)$ & $0(0-0)^{\mathrm{a}}$ & $3(1-3)^{b}$ & $3(2-3)^{b, c}$ & $<0.001^{\star \star \star}$ \\
\hline Mobility (0-3) & $0(0-0)^{\mathrm{a}}$ & $1(1-1)^{b}$ & $1(1-1)^{b, c}$ & $<0.001^{\star \star \star}$ \\
\hline OA score (0-192) & $0(0-2)^{a}$ & $10(7-16)^{b}$ & $14(9-19)^{b, c}$ & $<0.001^{\star \star \star}$ \\
\hline Joint pain score (0-48) & $0(0-0)^{\mathrm{a}}$ & $4(2-4)^{b}$ & $4(3-5)^{b, c}$ & $<0.001^{\star \star *}$ \\
\hline CBPI pain (0-10) & $0(0-0.0625)^{a}$ & $1.75(0-3.5)^{b}$ & $3.375(1.813-4.688)^{C}$ & $<0.001^{* \star *}$ \\
\hline CBPI function (0-10) & $0(0-0.833)^{a}$ & $1.167(0.1667-4.50)^{b}$ & $2.833(1.50-5.042)^{b, c}$ & $<0.001^{\star \star \star}$ \\
\hline HCPI (0-44) & $3(0-8.25)^{a}$ & $14(8-22)^{b}$ & $20.5(15.25-21.75)^{b, c}$ & $<0.001^{\star \star \star}$ \\
\hline ACVS stiffness (0-16) & $0(0-0.25)^{a}$ & $5(2-8)^{b}$ & $8(5-9)^{b, c}$ & $<0.001^{\star \star \star}$ \\
\hline ACVS function (0-16) & $0(0-0.25)^{\mathrm{a}}$ & $5(1-8)^{b}$ & $8(6-12)^{c}$ & $<0.001^{\star * \star}$ \\
\hline ACVS gait (0-20) & $0.5(0-2.25)^{\mathrm{a}}$ & $7(2-11)^{b}$ & $9(7-11.75)^{b, c}$ & $<0.001^{\star \star \star}$ \\
\hline ACVS QoL (0-12) & $0(0-1)^{\mathrm{a}}$ & $3(1-5)^{b}$ & $4.5(2.6)^{b, c}$ & $<0.001^{\star \star \star}$ \\
\hline $\operatorname{LOAD}(0-52)$ & $2(0-5)^{\mathrm{a}}$ & $14(9-23)^{b}$ & $18.5(12-23)^{b, c}$ & $<0.001^{\star \star *}$ \\
\hline Radiographic OA score (0-70) & $3(1-10)^{a}$ & $14(8.25-24.75)^{b}$ & $20(8-26)^{b, c}$ & $<0.001^{* * *}$ \\
\hline
\end{tabular}

Superscript letters indicate groupings within the data, shared superscripts indicate no significant difference between groups on post hoc testing, and differing superscripts indicate a difference with a $P$ value of less than 0.05 on post hoc testing. ${ }^{* \star \star} P \leq 0.001$

CBPI, Canine Brief Pain Inventory; HCPI, Helsinki Chronic Pain Index; LOAD, Liverpool Osteoarthritis in Dogs; OA, osteoarthritis; OANSAID, OA dogs receiving nonsteroidal anti-inflammatory drug.

The parameter estimates of those predictor variables significantly associated with the response are presented in Table 3. The final model, containing only the significant terms, demonstrated that the magnitude of the measured response increased as a curvilinear function of the stimulating current $(\mathrm{mA})$. There was a significant negative interaction between body weight and stimulating current (weight.mA; $P<0.001$ ); larger animals demonstrated a lesser increase in response magnitude with increasing current compared with smaller animals. There was a significant positive interaction between the OANSAID category and stimulating current (OANSAID.mA) compared with control $(P$ $<0.001)$ and OA category $(P<0.001)$ animals; OANSAID category animals demonstrated increased magnitude responses at a given stimulating current, compared with both control and $\mathrm{OA}$ category animals. These relationships are shown graphically in Figure 5, at a fixed weight of $25 \mathrm{~kg}$.

\subsubsection{Temporal summation early (A-fibre) response}

The magnitude of A-fibre responses increased with increasing stimulus number from 1 to 8 within each repetition of the protocol (TS) $(P<0.001)$ but was reduced on the third (final) occasion of the TS (train of 8) protocol, compared with the first $(P=0.013)$ (Table 4). Higher weight animals demonstrated reduced magnitude responses to stimulation $(P=0.001)$ and lesser increases in magnitude of response with increasing stimulus number (weight. stimulus number interaction) $(P=0.009)$. Osteoarthritis and OANSAID animals did not differ from control animals.

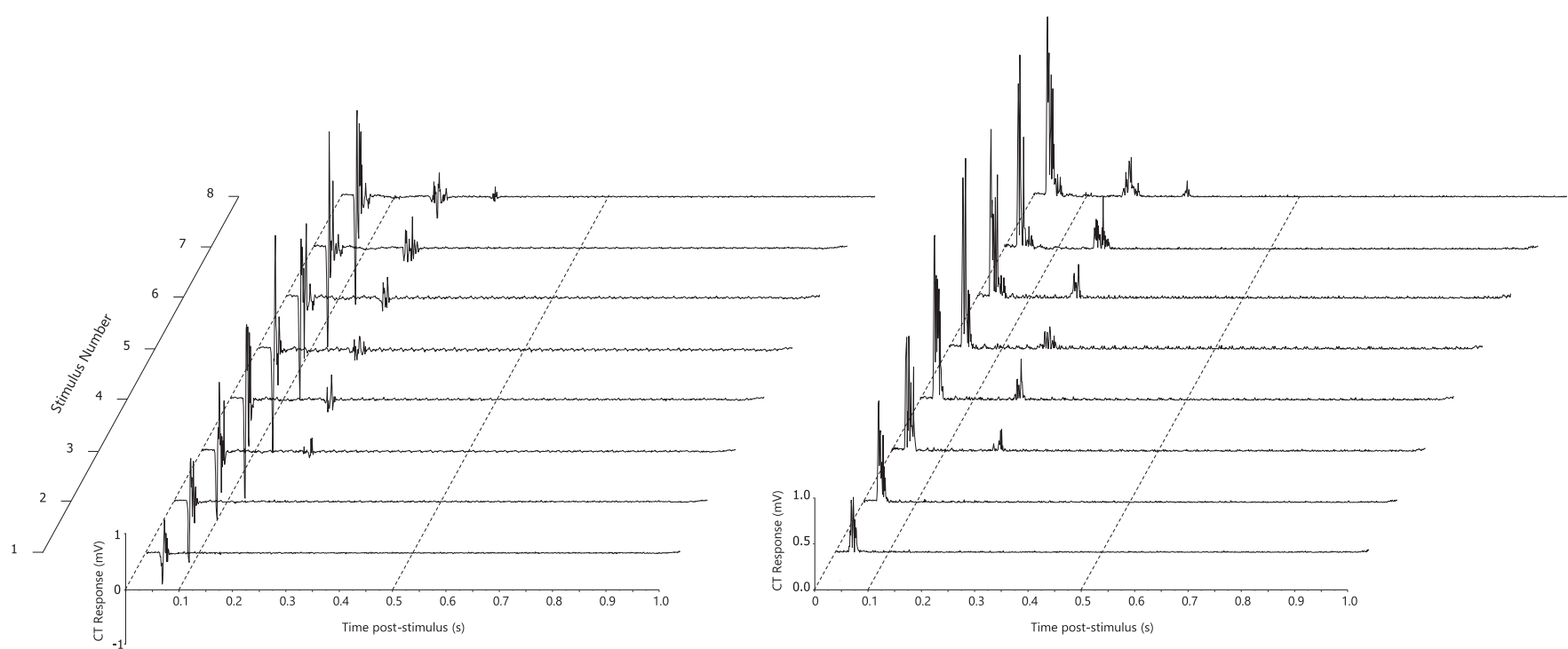

Figure 3. An example of temporal summation in the cranial tibial muscle (recorded from dog 71). The top channel is a stimulus marker channel, with a train of 8 , 1 -ms 10-mA stimuli delivered at a frequency of $1 \mathrm{~Hz}$. The lower channel shows the early and late responses in the cranial tibial muscle. The time base is 0.2 seconds/division. CT, cranial tibial. 


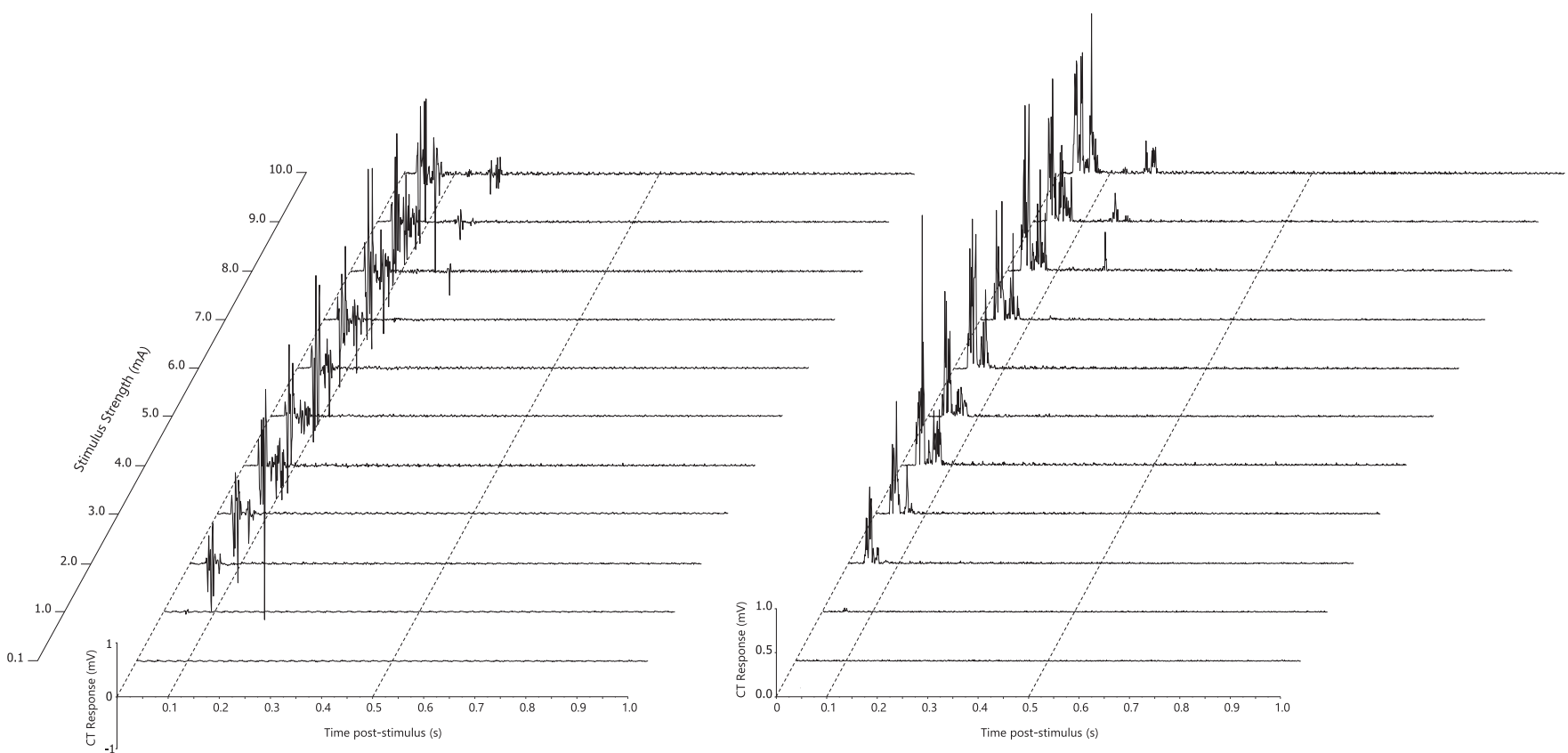

Figure 4. An example of the electrical stimulus response curve recorded from the cranial tibial muscle in dog 98 . The top channel is the stimulus marker channel, with each single line representing five 1-ms stimuli delivered at a frequency of $100 \mathrm{~Hz}$. Eleven stimuli were delivered with a 60 -second interval between them starting at $0.1 \mathrm{~mA}$ (baseline), $1 \mathrm{~mA}$, and increasing in $1 \mathrm{~mA}$ increments through to $10 \mathrm{~mA}$. The middle channel shows the early responses in the cranial tibial muscle, and the lower channel shows the rectified EMG response in the cranial tibial muscle. The time base is 0.2 seconds/division. CT, cranial tibial; EMG, electromyography.

\subsubsection{Temporal summation late (C-fibre) response}

The TS protocol consistently elicited late responses (Table 4). The magnitude of the late (C-fibre) response increased with increasing stimulus number from 1 to 8 within each repetition of the protocol (TS) $(P<0.001)$ but was decreased on both the second and third occasions of repeating the protocol (train of 8) compared with the first trial. Higher weight animals demonstrated lesser increases in magnitude of response with increasing stimulus number (weight.stimulus number interaction; $P<$ 0.001), and older animals also demonstrated lesser increases in magnitude of response with increasing stimulus number (age. stimulus number interaction; $P=0.001$ ). Both $\mathrm{OA}$ (OA.stimulus number interaction; $P<0.001$ ) and OANSAID (OANSAID. stimulus number interaction; $P=0.005)$ category animals demonstrated larger increases in magnitude of response with increasing stimulus number compared with control animals (Fig. 6), but there were no differences between the OA and OANSAID groups.

\subsection{Diffuse noxious inhibitory control investigation}

\subsubsection{Demographics}

Data were analysed from 12 control and 11 OA dogs (none receiving NSAIDs). The sex distribution between the groups was not different, and the distribution of breeds appeared well matched on visual inspection (Table 5). Osteoarthritis dogs were significantly older than control dogs (Table 5). Groups were not different in terms of weight; however, body condition score was

\section{Table 3}

Effect size estimates and $P$ values for the general linear model that was fitted to the stimulus response (early) data.

\begin{tabular}{|c|c|c|c|c|c|}
\hline & Response magnitude (mV/s) & SE & Conf int $2.5 \%$ & Conf int $97.5 \%$ & $P$ \\
\hline Intercept & -0.001230 & 0.003234 & -0.007569 & 0.005109 & 0.704 \\
\hline Weight & 0.000018 & 0.000113 & -0.000204 & 0.000240 & 0.873 \\
\hline $\mathrm{OA}$ & 0.000753 & 0.002481 & -0.004110 & 0.005615 & 0.762 \\
\hline OANSAID & 0.000353 & 0.002782 & -0.005100 & 0.005806 & 0.899 \\
\hline $\mathrm{mA}$ & 0.004864 & 0.000540 & 0.003807 & 0.005922 & $<0.001^{\star \star \star}$ \\
\hline$m A^{2}$ & -0.000170 & 0.000052 & -0.000271 & -0.000069 & $0.001^{\star \star}$ \\
\hline weight.mA & -0.000094 & 0.000019 & -0.000132 & -0.000056 & $<0.001^{\star \star \star}$ \\
\hline weight. $\mathrm{mA}^{2}$ & 0.000004 & 0.000002 & 0.000001 & 0.000008 & $0.026^{*}$ \\
\hline $\mathrm{OA} \cdot \mathrm{mA}$ & -0.000092 & 0.000119 & -0.000325 & 0.000141 & 0.440 \\
\hline OANSAID.mA & 0.000759 & 0.000134 & 0.000497 & 0.001021 & $<0.001^{\star \star \star}$ \\
\hline
\end{tabular}

${ }^{*} P \leq 0.05 ;{ }^{* \star} P \leq 0.01 ;{ }^{* \star} P \leq 0.001$.

$\mathrm{OA}$, osteoarthritis; OANSAID, OA dogs receiving nonsteroidal anti-inflammatory drug. 


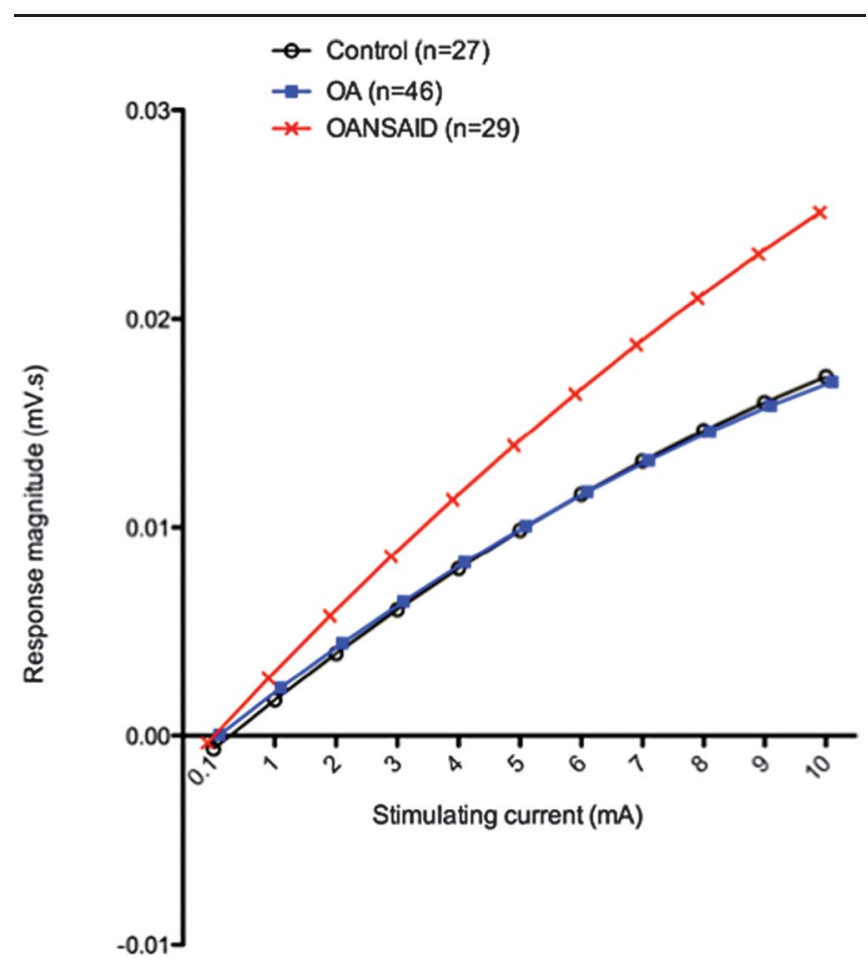

Figure 5. Illustration of the mean curves predicted by the general linear model for stimulus response of dogs within differing OA categories, assuming a weight of $25 \mathrm{~kg}$. Each data point for the control animals is based on $27 \mathrm{dogs}$; for the OA group, it is based on 46 dogs; and for the OANSAID group, it is based on 29 dogs. For each animal, the mean response to the 2 repetitions of the stimulus response curve was averaged before analysis. The y-axis represents the natural logarithm of the magnitude of the EMG response, and the $x$-axis shows the magnitude of the stimulating current. EMG, electromyography; OA, osteoarthritis; OANSAID, OA dogs receiving nonsteroidal antiinflammatory drug.

higher in OA $(6,5-7)$ compared with control dogs (5, 4.25-5.75, $P=0.047)$.

\subsubsection{Veterinary musculoskeletal and gait assessments}

Degree of lameness, mobility impairment, OA burden, and joint pain burden were all increased in OA compared with control dogs (Table 6).

\subsubsection{Owner-completed clinical metrology instruments}

The CBPI, Helsinki Chronic Pain Index, ACVS Canine Orthopaedic Index, and LOAD were all rated significantly higher by owners of OA compared with control dogs (Table 6), but there was no significant difference in scores for the SNoRE questionnaire.

\subsubsection{Radiography}

Significantly more radiographic signs of OA were identified in dogs in the OA compared with control group, and significantly more of the 7 joints assessed demonstrated radiographic signs of OA in OA compared with control dogs (Table 6).

\subsubsection{Nociceptive withdrawal reflex threshold}

The threshold current required to elicit an NWR was significantly higher in OA (3.8 [95\% Cl 2.4-5.2 mA]) compared with control dogs (1.9 [95\% Cl 1.4-2.5 mA], $P=0.013$ ) (Table 6).

\subsubsection{Diffuse noxious inhibitory control efficacy}

The 2xThr stimulation did not elicit consistent late responses, therefore only the early $(0-100 \mathrm{~ms})$ latency response was analysed. $^{19}$

The final significant general linear model that described the magnitude of the early response took the form of an equation, the parameter estimates of which and $P$ values associated with the predictor variables within the model are presented in Table 7. The predictor variables and their relationship with the magnitude of the response are described below. Time and age were considered continuous scale variables. Each occasion of DNIC testing (pre, DNIC 1, DNIC 2, and post) was considered a categorical variable, as was $\mathrm{OA}$ status (OA/control). Figure 7 shows the effect of mechanical "conditioning" stimulation of the forepaw on electrically evoked "test" EMG reflexes in the CT muscle of the contralateral hind limb.

\subsubsection{Stability of response magnitude within occasion}

Time alone did not account for a significant variation in magnitude within a test occasion $(P=0.069)$.

\subsubsection{Stability of response magnitude between occasions}

Between different test occasions, response magnitude was decreased in DNICs 1 and 2, and in the post-DNIC state, compared with the original pre-DNIC occasion $(P=0.048$, $<0.001$, and $<0.001$ respectively), indicating a decreasing magnitude of response with repeated occasions of the stimulating protocol.

\subsubsection{Efficacy of diffuse noxious inhibitory control stimulus}

There was a significant interaction between time and occasion for DNICs 1 and $2(P<0.001)$, but not between time and occasion post-DNIC $(P=0.50)$, demonstrating that the application of the conditioning stimulus was responsible for significantly decreasing the response magnitude during DNICs 1 and 2 compared with the pre-DNIC occasion. The interaction between square and cubic terms of time, and DNICs 1 and 2, was significant, indicating a curvilinear change of response with application of the conditioning stimulus.

\subsubsection{Effect of osteoarthritis status}

Osteoarthritis status alone had no significant effect on response magnitude $(P=0.31)$; however, there was a significant interaction between $\mathrm{OA}$ status and occasion during the DNIC 2 $(P=0.003)$ and post-DNIC $(P=0.02)$ testing, which predicted a higher magnitude of response (ie, decreased inhibition of response) in OA dogs during these 2 occasions, compared with control dogs. Inclusion of the overall interaction between $\mathrm{OA}$ status and DNIC occasion as a predictor variable significantly improved the model (change in log likelihood $=7.82$, df3; $P=$ 0.0499).

\subsubsection{Effect of age}

The effect of age was tested within models but found to be not significant as either a main effect within the model, nor in interaction with other terms within the models.

\section{Discussion}

These studies have shown that several characteristics of the CT NWR were altered in dogs with OA, therefore central neurophysiological changes may play a role in the pathology 
Table 4

Effect size estimates and $\boldsymbol{P}$ values for the general linear model that was fitted to the temporal summation data.

\begin{tabular}{|c|c|c|c|c|c|c|c|c|c|c|}
\hline & $\begin{array}{l}\text { Temporal summation early } \\
\text { response (InmV/s) }\end{array}$ & SE & $\begin{array}{l}\text { Conf int } \\
2.5 \%\end{array}$ & $\begin{array}{l}\text { Conf int } \\
97.5 \%\end{array}$ & $P$ & $\begin{array}{l}\text { Temporal summation late } \\
\text { response (InmV/s) }\end{array}$ & SE & $\begin{array}{l}\text { Conf int } \\
2.5 \%\end{array}$ & $\begin{array}{l}\text { Conf int } \\
97.5 \%\end{array}$ & $P$ \\
\hline Intercept & -4.900 & 0.341 & -5.569 & -4.231 & $<0.001^{\star \star \star}$ & -7.142 & 0.700 & -8.513 & -5.771 & $<0.001^{*}$ \\
\hline Weight & -0.040 & 0.012 & -0.064 & -0.017 & $0.001^{\star \star}$ & 0.017 & 0.015 & -0.012 & 0.046 & 0.246 \\
\hline $\mathrm{OA}$ & & & & & & -0.722 & 0.348 & -1.404 & -0.040 & $0.038^{*}$ \\
\hline OANSAID & & & & & & -0.254 & 0.362 & -0.964 & 0.456 & 0.483 \\
\hline Occasion 2 & -0.022 & 0.019 & -0.059 & 0.016 & 0.265 & -0.058 & 0.026 & -0.109 & -0.007 & $0.026^{*}$ \\
\hline Occasion 3 & -0.048 & 0.019 & -0.086 & -0.010 & $0.013^{\star}$ & -0.120 & 0.026 & -0.171 & -0.069 & $<0.001^{\star \star \star}$ \\
\hline $\begin{array}{l}\text { Stimulus } \\
\text { number }\end{array}$ & 1.084 & 0.145 & 0.800 & 1.369 & $<0.001^{\star \star \star}$ & 2.401 & 0.373 & 1.670 & 3.132 & $<0.001^{\star \star \star}$ \\
\hline $\begin{array}{l}\text { Stimulus } \\
\text { number }^{2}\end{array}$ & -0.243 & 0.036 & -0.314 & -0.171 & $<0.001^{\star \star *}$ & -0.474 & 0.094 & -0.658 & -0.291 & $<0.001^{\text {***}}$ \\
\hline $\begin{array}{l}\text { Stimulus } \\
\text { number }^{3}\end{array}$ & 0.016 & 0.003 & 0.011 & 0.021 & $<0.001^{\star \star \star}$ & 0.030 & 0.007 & 0.016 & 0.043 & $<0.001^{* * *}$ \\
\hline $\begin{array}{l}\text { Weight. } \\
\text { stimulus } \\
\text { number }\end{array}$ & -0.014 & 0.005 & -0.024 & -0.003 & $0.009^{\star *}$ & -0.037 & 0.008 & -0.053 & -0.022 & $<0.001^{\star \star \star}$ \\
\hline $\begin{array}{l}\text { Weight. } \\
\text { stimulus } \\
\text { number }\end{array}$ & 0.004 & 0.001 & 0.001 & 0.006 & $0.006^{\star \star}$ & 0.008 & 0.002 & 0.004 & 0.012 & $<0.001^{\star \star \star}$ \\
\hline \multirow{11}{*}{$\begin{array}{l}\text { Weight. } \\
\text { stimulus } \\
\text { number }^{3}\end{array}$} & 0.000 & 0.000 & 0.000 & 0.000 & $0.009^{\star \star}$ & -0.001 & 0.000 & -0.001 & 0.000 & $<0.001^{\star \star \star}$ \\
\hline & OA.stimulus number & & - & - & - & 0.805 & 0.186 & 0.442 & 1.169 & $<0.001^{\star \star \star}$ \\
\hline & OANSAID.stimulus number & & - & - & - & 0.540 & 0.193 & 0.161 & 0.919 & $0.005^{\star \star}$ \\
\hline & OA.stimulus number ${ }^{2}$ & & - & - & - & -0.160 & 0.047 & -0.251 & -0.069 & $0.001^{\star *}$ \\
\hline & OANSAID.stimulus number ${ }^{2}$ & & - & - & - & -0.100 & 0.048 & -0.195 & -0.005 & $0.039^{\star}$ \\
\hline & OA.stimulus number ${ }^{3}$ & & - & - & - & 0.010 & 0.003 & 0.004 & 0.017 & $0.003^{\star \star}$ \\
\hline & OANSAID.stimulus number ${ }^{3}$ & & - & - & - & 0.006 & 0.004 & -0.001 & 0.013 & 0.079 \\
\hline & Age & & - & - & - & 0.088 & 0.067 & -0.043 & 0.218 & 0.187 \\
\hline & Age.stimulus number & & - & - & - & -0.121 & 0.036 & -0.190 & -0.051 & $0.001^{* *}$ \\
\hline & Age.stimulus number ${ }^{2}$ & & - & - & - & 0.024 & 0.009 & 0.007 & 0.042 & $0.006^{\star \star}$ \\
\hline & Age.stimulus number ${ }^{3}$ & & - & - & - & -0.002 & 0.001 & -0.003 & 0.000 & $0.016^{*}$ \\
\hline
\end{tabular}

${ }^{\star} P \leq 0.05 ;{ }^{* \star} P \leq 0.01 ;{ }^{\star \star \star} P \leq 0.001$.

OA, osteoarthritis; OANSAID, OA dogs receiving nonsteroidal anti-inflammatory drug.

of OA-associated pain and disability in dogs. Diffuse noxious inhibitory control investigations suggest that these central changes may be related in part to less effective descending inhibition of nociceptive stimuli.

In man, the RIII (A $\delta$-fibre-mediated) threshold is correlated with the pain threshold ${ }^{35}$ and is decreased in painful OA states. ${ }^{12} \mathrm{We}$ anticipated that dogs exhibiting CS would demonstrate a diminished threshold to elicit an NWR; however, our results indicated that threshold current was higher in OA animals compared with controls. The underlying reason for this finding is difficult to explain. The early latency $(0-100 \mathrm{~ms})$ response elicited by NWR stimulation in our testing paradigm comprises both $A \beta$ (RII equivalent in man) and $A \delta$ (RIII equivalent) transmission. The RII response in man is considered non-nociceptive and elicited by subpain threshold intensities of stimulation. Central sensitisation may be accompanied by hypoaesthesia to one or more sensory modalities in human subjects, ${ }^{18}$ therefore it is possible that the greater threshold identified in OA dogs relates to $A \beta$-mediated hypoaesthesia. Although it may have been desirable to further divide the responses by latency into $A \beta$ - or $A \delta$ mediated, as reported by Bergadano et al., 6 we undertook testing in a mixed population of dogs with a range of weights and conformations, which would have added to the variability in response latency. Visual inspection of pilot data traces revealed that we could only consistently identify an early (A-fibre) and late (C-fibre) response. ${ }^{19}$ We could have considered measuring the afferent distance of the conduction pathway in individual animals and using this, together with an estimate of conduction velocity, to calculate more accurately the latency window of the NWR in each individual dog. However, our inclusion criteria for the study limited the weight range of the dogs included in the study, therefore this was not deemed necessary for the present investigation.

The stimulus response curve demonstrated facilitation of the early response in OANSAID dogs, compared with both control and OA dogs. The amplitude of the RIII response has been shown to correlate with the magnitude of subjective pain in conscious human volunteers ${ }^{13}$; therefore, the inference from our data is that 


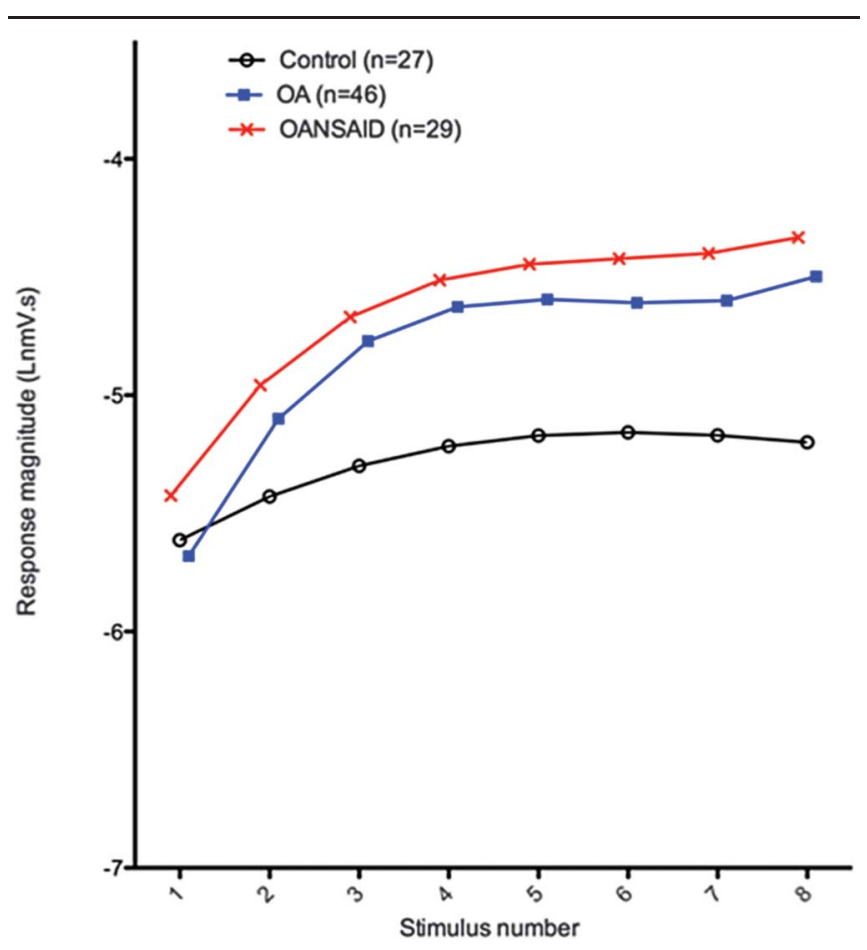

Figure 6. Illustration of the mean curves predicted by the general linear model for the first occasion temporal summation late response for dogs within differing $\mathrm{OA}$ categories, assuming a weight of $25 \mathrm{~kg}$ and age of 9 years. The $y$-axis represents the natural logarithm of the magnitude of the EMG response, and the $x$-axis shows stimulus number. EMG, electromyography; OA, osteoarthritis; OANSAID, $\mathrm{OA}$ dogs receiving nonsteroidal anti-inflammatory drug.

OANSAID dogs may exhibit hyperalgesia, compared with dogs in both the $\mathrm{OA}$ and control groups. The fact that the $\mathrm{OA}$ and OANSAID groups were not different based on veterinarian examination scores and radiographic OA scores were not unexpected-there are no validated veterinarian assessment systems of $O A$ pain, and radiographic evidence of $O A$ is known not to be correlated with pain, just as in humans. Although OA and OANSAID groups were comparable with respect to the majority of the clinical metrology instrument data, OANSAIDs

\section{Table 5}

\section{Demographic data.}

\begin{tabular}{|c|c|c|c|}
\hline & Control $(n=12)$ & $O A(n=11)$ & $P$ \\
\hline \multicolumn{4}{|l|}{ Breed } \\
\hline Labrador & 6 & 3 & - \\
\hline Collie & 2 & 1 & - \\
\hline Retriever & 2 & 2 & - \\
\hline Lurcher & 2 & 1 & - \\
\hline German Shepherd & 0 & 1 & - \\
\hline Rottweiler & 0 & 2 & - \\
\hline Spaniel & 0 & 1 & - \\
\hline \multicolumn{4}{|l|}{ Sex } \\
\hline Male neuter & 6 & 5 & 1.0 \\
\hline Female neuter & 6 & 6 & 1.0 \\
\hline Weight & $\begin{array}{l}23.8(95 \% \\
\text { Cl 21.6-26.1) }\end{array}$ & $\begin{array}{l}31.3(95 \% \\
\text { Cl 23.2-39.4) }\end{array}$ & 0.053 \\
\hline Age & $\begin{array}{l}7.5(95 \% \\
\text { Cl 6.9-8.2) }\end{array}$ & $\begin{array}{l}9.8(95 \% \\
\mathrm{Cl} 8.5-11.1)\end{array}$ & $0.002^{* *}$ \\
\hline Body condition score (0-9) & $5(4.25-5.75)$ & $6(5-7)$ & $0.047^{*}$ \\
\hline
\end{tabular}

$\mathrm{OA}$, osteoarthritis. were significantly more affected with respect to the CBPI pain and the ACVS description of function subscales and had higher scores on all the other validated clinical metrology instruments (LOAD and CBPI function). These data indicate that the OANSAID group was more severely affected by OA pain and suggest that treatment with commonly prescribed veterinary NSAIDs ${ }^{20}$ may not prevent or reverse CS, despite the tentative conclusion from a recent study in humans with $\mathrm{OA}$ investigating etoricoxib. ${ }^{1}$ The total duration of treatment with NSAIDs in the OANSAID group was not recorded in individual dogs in this study, and it is possible that differences in the duration of administration introduced variability into the data. However, all dogs in the OANSAID group had been receiving NSAIDs for at least 3 months before recruitment to the study which, from early data in humans, ${ }^{1}$ would be sufficient time for the NSAID to exhibit an antihyperalgesic effect.

Temporal summation data demonstrated no group differences for the early (A-fibre-mediated) response but facilitation of the late (mostly C-fibre) response in OA and OANSAID dogs, compared with controls. The absence of an effect on the early response data is likely due to a significant component being mediated by lowthreshold A-beta fibres. The applied 10-mA stimulus, designed as a suprathreshold stimulus, would cause the early response to saturate at this level of stimulation, and therefore, differences between groups were minimised. By contrast, the higherthreshold C-fibre-mediated late response displayed the expected increasing magnitude with repeated stimuli and, in alignment with our hypothesis, was augmented in both OA and OANSAID groups compared with the control group. This likely indicates that $\mathrm{OA}$ is associated with CS in dogs. It is also possible that the EMG findings for C-fibre-mediated responses are due to C-fiber sensitisation rather than CS, although it is difficult to make a distinction between these 2 effects in our data set.

The data produced during the DNIC investigation demonstrate both that MCS elicits quantifiable DNIC in anaesthetised dogs, and that the efficacy of DNIC is compromised in dogs with OA, compared with a control group. A recent meta-analysis concluded that, despite methodological limitations, a number of chronic pain conditions in man, including $O A$, are associated with reduced efficacy of CPM. ${ }^{28}$ Reduced net efficacy of nociceptive inhibition may arise through impaired descending antinociceptive modulation or through descending facilitation of nociceptive signalling. ${ }^{3}$ We did not probe each of these pathways independently in these clinical cases; however, the magnitude of measured EMG response in this study represents the net effect of balance between inhibitory and facilitatory mechanisms; therefore, these data provide evidence that the balance of descending pathways becomes shifted toward pronociception in canine OA.

The differences between $\mathrm{OA}$ and control groups were only evident on DNIC 2, and then persisted into the post-DNIC period. Because previous data on DNIC in dogs using MCS were not available, numbers required to identify significant differences were unknown; however, it is clear from our results that the interaction between group and occasion begins to approach significance during DNIC $1(P=0.07)$. Had larger sample sizes been used, we would have had greater power to detect differences between groups, and may have identified a significant difference during DNIC 1. The small sample size is a major limitation of the DNIC investigation and reflected difficulties in establishing the methodology to elicit DNIC in dogs. Only 5 minutes was allowed to elapse between the TS protocol and the start of the DNIC investigation. This time period was kept deliberately short to avoid prolonging the anaesthesia time for the dogs as far as possible. It is possible that delivery of 


\section{Table 6}

Musculoskeletal examination, owner-completed metrology instrument, radiographic scoring, and nociceptive withdrawal reflex (NWR) data in dogs undergoing the DNIC protocol.

\begin{tabular}{|c|c|c|c|}
\hline & Control & OA & $P$ \\
\hline Lameness $(0-10)$ & $0(0-0)$ & $3(3-3)$ & $<0.001^{\star \star \star}$ \\
\hline Mobility (0-3) & $0(0-0)$ & $1(1-2)$ & $<0.001^{\star \star \star}$ \\
\hline OA score (0-192) & $0(0-2)$ & $9(6-12)$ & $<0.001^{\star \star \star}$ \\
\hline Joint pain score (0-48) & $0(0-0)$ & $4(2-5)$ & $<0.001^{\star \star \star}$ \\
\hline CBPI pain $(0-10)$ & $0(0-0)$ & $1.125(0-2.69)$ & $0.0085^{\star \star}$ \\
\hline CBPI function (0-10) & $0(0-0)$ & $2.375(0-6.938)$ & $0.0022^{\star \star}$ \\
\hline HCPI (0-44) & $1(0-1.75)$ & $15.5(3.5-20.5)$ & $0.0026^{\star *}$ \\
\hline ACVS stiffness (0-16) & $0(0-0)$ & $5.5(0-7)$ & $0.0029^{\star \star}$ \\
\hline ACVS function (0-16) & $0(0-0)$ & $4(0-8.75)$ & $0.0076^{\star *}$ \\
\hline ACVS gait (0-20) & $0(0-0)$ & $5(2.25-11.5)$ & $0.0022^{\star *}$ \\
\hline ACVS QoL (0-12) & $0(0-0.75)$ & $3(0-6.25)$ & $0.0076^{\star *}$ \\
\hline $\operatorname{LOAD}(0-52)$ & $2.5(0-3)$ & $15.5(5-25)$ & $0.0042^{\star \star}$ \\
\hline SNoRE & $13.5(10.5-18.5)$ & $15.5(14-25.25)$ & 0.21 \\
\hline Radiographic OA score (0-70) & $2(0.25-3)$ & $20(16-28)$ & $<0.001^{\star \star \star}$ \\
\hline Number of joints radiographically affected & $1(0.25-2)$ & $5(2-6)$ & $<0.001^{\star * \star}$ \\
\hline NWR threshold & $1.9(95 \% \mathrm{Cl} 1.4-2.5)$ & 3.8 (95\% Cl 2.4-5.2) & $0.013^{*}$ \\
\hline
\end{tabular}

${ }^{*} P \leq 0.05 ;{ }^{* *} P \leq 0.01 ;{ }^{* *} P \leq 0.001$.

CBPI, Canine Brief Pain Inventory; Cl, confidence interval; DNIC, diffuse noxious inhibitory control; HCPI, Helsinki Chronic Pain Index; LOAD, Liverpool Osteoarthritis in Dogs; OA, osteoarthritis; SNoRE, sleep and night time restlessness evaluation.

\section{Table 7}

Parameter estimates, SE, $95 \%$ Cls, and $\boldsymbol{P}$ values for the general linear model fitted to the stimulus response (early) data $(\ln (\mathrm{mV} / \mathrm{s}))$.

\begin{tabular}{|c|c|c|c|c|c|}
\hline Predictor variable & Parameter estimate & SE & Conf int $2.5 \%$ & Conf int $\mathbf{9 7 . 5 \%}$ & $P$ \\
\hline \multicolumn{6}{|l|}{ Fixed effects } \\
\hline Cons & -5.420132 & 0.265083 & -5.939685 & -4.90058 & $<0.001^{\star \star \star}$ \\
\hline DNIC 1 & -0.158861 & 0.095373 & -0.345789 & 0.028067 & $0.048^{*}$ \\
\hline DNIC 2 & -0.508912 & 0.095373 & -0.695839 & -0.321984 & $<0.001^{\star \star \star}$ \\
\hline Post-DNIC & -0.433574 & 0.100943 & -0.631419 & -0.235729 & $<0.001^{\star \star \star}$ \\
\hline Time & -0.009741 & 0.006579 & -0.022635 & 0.003153 & 0.069 \\
\hline Time $^{2}$ & -0.000054 & 0.000418 & -0.000873 & 0.000764 & 0.448 \\
\hline Time $^{3}$ & 0.000005 & 0.000007 & -0.00001 & 0.000019 & 0.265 \\
\hline $\mathrm{OA}$ & -0.183357 & 0.381486 & -0.931054 & 0.564341 & 0.315 \\
\hline OA.DNIC 1 & 0.181664 & 0.127397 & -0.068029 & 0.431357 & 0.077 \\
\hline OA.DNIC 2 & 0.349945 & 0.127397 & 0.100251 & 0.599638 & $0.003^{\star *}$ \\
\hline OA.post-DNIC & 0.271047 & 0.131377 & 0.013553 & 0.528541 & $0.020^{\star *}$ \\
\hline Time.DNIC 1 & -0.055631 & 0.009303 & -0.073866 & -0.037397 & $<0.001^{\star \star \star}$ \\
\hline Time $^{2}$.DNIC 1 & 0.003449 & 0.000591 & 0.002291 & 0.004607 & $<0.001^{\text {***}}$ \\
\hline Time $^{3}$.DNIC 1 & -0.000052 & 0.00001 & -0.000072 & -0.000032 & $<0.001^{\star \star \star}$ \\
\hline Time.DNIC 2 & -0.05043 & 0.009303 & -0.068664 & -0.032195 & $<0.001^{\star \star \star}$ \\
\hline Time $^{2}$.DNIC 2 & 0.00353 & 0.000591 & 0.002372 & 0.004688 & $<0.001^{\star \star \star}$ \\
\hline Time $^{3}$.DNIC 2 & -0.000057 & 0.00001 & -0.000077 & -0.000037 & $<0.001^{\star \star \star}$ \\
\hline Time.post-DNIC & 0.000054 & 0.009522 & -0.01861 & 0.018717 & 0.497 \\
\hline Time $^{2}$.post-DNIC & 0.000264 & 0.000605 & -0.000922 & 0.001449 & 0.331 \\
\hline Time $^{3}$.post-DNIC & -0.000006 & 0.00001 & -0.000026 & 0.000015 & 0.299 \\
\hline
\end{tabular}

${ }^{*} P \leq 0.05 ;{ }^{* \star} P \leq 0.01 ;{ }^{* \star} P \leq 0.001$.

$\mathrm{Cl}$, confidence interval; DNIC, diffuse noxious inhibitory control; $\mathrm{OA}$, osteoarthritis. 


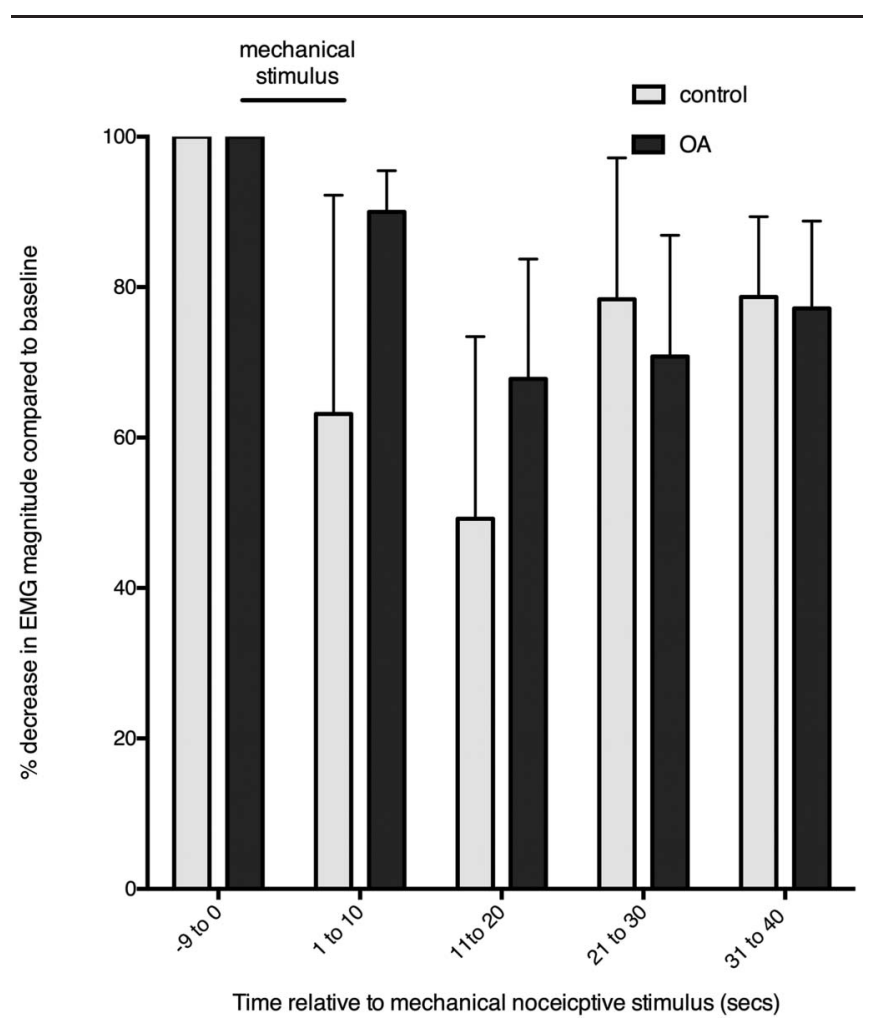

Figure 7. Effect of mechanical "conditioning" stimulation of the forepaw on electrically evoked "test" EMG reflexes in the cranial tibial muscle of the contralateral hind limb. Clip was applied at time 0 for 20 seconds. In the control group, EMG responses to the test stimulus were reduced (greater \% reduction in EMG) during clip application, indicating antinociception and a DNIC effect. When all time points were considered, DNIC in the OA group $(n=11)$ was significantly less compared with control animals $(n=12)(P=0.016)$. Responses are medians, errors are 75th percentiles. DNIC, diffuse noxious inhibitory control; EMG, electromyography; OA, osteoarthritis.

a supramaximal stimulus during the TS protocol sensitised the nociceptive system so that the nociceptive pathways were not in a naive state at the start of the DNIC experiment, and this may have affected our DNIC results. The optimal time delay between TS and measurement of DNIC is currently unknown.

Nociceptive withdrawal reflexes are segmental spinal reflexes, subject to supraspinal modulation. ${ }^{11}$ Alfaxalone anaesthesia enabled NWR recording in client-owned dogs. While alfaxalone increases NWR threshold and decreases magnitude of response to electrical stimulation, ${ }^{19}$ there is no reason to expect a differential effect of the anaesthetic on control vs OA or OANSAID animals, as alfaxalone is devoid of analgesic activity. ${ }^{39}$

Regarding assessment of DNIC, many sedatives and analgesics will interact with descending pronociceptive and antinociceptive pathways ${ }^{27,36}$ and could alter the measured responses. Acepromazine has been shown not to modulate $\mathrm{NWR}^{7}$ and, given it is considered to have no antinociceptive properties, ${ }^{4}$ would not be expected to interact with descending modulatory mechanisms. Alfaxalone is a gamma aminobutyric acid agonist, and DNIC is reportedly unaffected by gamma aminobutyric acid agonists, ${ }^{23}$ therefore we consider that the form of anaesthesia used was appropriate to our investigation.

Although we have identified group-level differences in DNIC efficacy, the aim is ultimately to identify individuals in which decreased DNIC efficacy contributes to the pain phenotype and address this mechanism therapeutically. ${ }^{3}$ Determining a normal "range" of DNIC responses in dogs will require the study of additional numbers of dogs of a wider demographic, particularly in view of the inconsistently reported sex $^{32}$ and age ${ }^{25}$ differences associated with CPM in man.

In conclusion, we have demonstrated a number of neurophysiological changes indicative of CS processes in dogs affected by spontaneous OA, consistent with findings in man. However, measurement of electrical thresholds seemed not to be a suitable parameter for CS using the current methods. The mechanisms involved may encompass both upregulation of nociceptive afferent pathways, ${ }^{26}$ in addition to alterations in the balance of descending modulatory mechanisms as shown here. Increasingly, it seems that the pathophysiological mechanisms of human $\mathrm{OA}^{21}$ are shared by the spontaneous disease in dogs, further validating canine spontaneous $\mathrm{OA}$ as a model for the human disease ${ }^{31,38}$ and supporting the use of dogs for mechanistic clinical trials to advance therapeutic development in humans.

\section{Conflict of interest statement}

The authors have no conflict of interest to declare.

This study was supported by the BBSRC grant number BB/ L00240X/1. The alfaxalone was supplied by Jurox (United Kingdom) Limited. J.R. Hunt, H. Jenkins, and M. Goff positions were funded by the BBSRC Grant number BB/L00240X/1.

\section{Acknowledgements}

The authors are grateful to Lindsey Crane and Liz Carthey, both of the Small Animal Hospital, University of Bristol, for undertaking the radiography procedures.

\section{Appendix A. Supplemental digital content}

Supplemental digital content associated with this article can be found online at http://links.Iww.com/PAIN/A622.

\section{Article history:}

Received 16 December 2017

Received in revised form 8 June 2018

Accepted 2 July 2018

Available online 9 July 2018

\section{References}

[1] Arendt-Nielsen L, Egsgaard LL, Petersen KK. Evidence for a central mode of action for etoricoxib (COX-2 inhibitor) in patients with painful knee osteoarthritis. PAIN 2016;157:1634-44.

[2] Arendt-Nielsen L, Nie H, Laursen MB, Laursen BS, Madeleine P, Simonsen $\mathrm{OH}$, Graven-Nielsen T. Sensitization in patients with painful knee osteoarthritis. PAIN 2010;149:573-81.

[3] Bannister K, Patel R, Goncalves L, Townson L, Dickenson AH. Diffuse noxious inhibitory controls and nerve injury: restoring an imbalance between descending monoamine inhibitions and facilitations. PAIN 2015; 156:1803-11.

[4] Barnhart MD, Hubbell JAE, Muir WW. Evaluation of the analgesic properties of acepromazine maleate, oxymorphone, medetomidine and a combination of acepromazine-oxymorphone. Vet Anaes Analg 2000; 27:89-96.

[5] Bars DL, Dickenson AH, Besson JM. Diffuse noxious inhibitory controls (DNIC). II. Lack of effect on non-convergent neurones, supraspinal involvement and theoretical implications. PAIN 1979;6:305-27.

[6] Bergadano A, Andersen OK, Arendt-Nielsen L, Schatzmann U, Spadavecchia C. Quantitative assessment of nociceptive processes in conscious dogs by use of the nociceptive withdrawal reflex. Am J Vet Res 2006;67:882-9.

[7] Bergadano A, Andersen OK, Arendt-Nielsen L, Spadavecchia C. Modulation of nociceptive withdrawal reflexes evoked by single and repeated nociceptive stimuli in conscious dogs by low-dose acepromazine. Vet Anaes Analg 2009;36:261-72. 
[8] Bergadano A, Andersen OK, Arendt-Nielsen L, Spadavecchia C. Noninvasive assessment of the facilitation of the nociceptive withdrawal reflex by repeated electrical stimulations in conscious dogs. Am J Vet Res 2007;68:899-907.

[9] Brown DC. The Canine Orthopedic Index. Step 3: responsiveness testing. Vet Surg 2014;43:247-54

[10] Brown DC, Boston RC, Coyne JC, Farrar JT. Development and psychometric testing of an instrument designed to measure chronic pain in dogs with osteoarthritis. Am J Vet Res 2007;68:631-7.

[11] Clarke RW, Harris J. The organization of motor responses to noxious stimuli. Brain Res Rev 2004;46:163-72.

[12] Courtney CA, Lewek MD, Witte PO, Chmell SJ, Hornby TG. Heightened flexor withdrawal responses in subjects with knee osteoarthritis. J Pain 2009;10:1242-9.

[13] Dowman R. Spinal and supraspinal correlates of nociception in man. PAIN 1991;45:269-81.

[14] Grosen K, Vase L, Pilegaard HK, Pfeiffer-Jensen M, Drewes AM. Conditioned pain modulation and situational pain catastrophizing as preoperative predictors of pain following chest wall surgery: a Prospective Observational Cohort Study. PLoS One 2014;9:e90185.

[15] Harris J. Involvement of spinal $\alpha 2$-adrenoceptors in prolonged modulation of hind limb withdrawal reflexes following acute noxious stimulation in the anaesthetised rabbit. Eur J Neurosci 2016;43:834-45.

[16] Harris LK. Mechanical nociceptive testing in dogs with osteoarthritis [PhD thesis]: University of Bristol, United Kingdom, 2016.

[17] Hielm-Bjorkman AK, Rita H, Tulamo RM. Psychometric testing of the Helsinki Chronic Pain Index by completion of a questionnaire in Finnish by owners of dogs with chronic signs of pain caused by osteoarthritis. Am J Vet Res 2009;70:727-34.

[18] Hochman JR, Davis AM, Elkayam J, Gagliese L, Hawker GA. Neuropathic pain symptoms on the modified painDETECT correlate with signs of central sensitization in knee osteoarthritis. Osteoarthr Cartil 2013;21:1236-42.

[19] Hunt J, Murrell J, Knazovicky D, Harris J, Kelly S, Knowles TG, Lascelles BDX. Alfaxalone anaesthesia facilitates electrophysiological recordings of nociceptive withdrawal reflexes in dogs (Canis familiaris). PLoS One 2016;11:e0158990.

[20] Hunt JR, Dean RS, Davis GND, Murrell JC. An analysis of the relative frequencies of reported adverse events associated with NSAID administration in dogs and cats in the United Kingdom. Vet J 2015; 206:183-90.

[21] Knazovicky D, Helgeson ES, Case B, Gruen ME, Maixner W, Lascelles BDX. Widespread somatosensory sensitivity in naturally occurring canine model of osteoarthritis. PAIN 2016;157:1325-32.

[22] Knazovicky D, Tomas A, Motsinger-Reif A, Lascelles BDX. Initial evaluation of nighttime restlessness in a naturally occurring canine model of osteoarthritis pain. PeerJ 2015;3:e772.

[23] Kunz M, Scholl KE, Schu U, Lautenbacher S. GABAergic modulation of diffuse noxious inhibitory controls (DNIC): a test by use of lorazepam. Exp Brain Res 2006;175:363-71.

[24] Laflamme D. Development and validation of a body condition score system for dogs. Santa Barbara: Canine practice, 1997.
[25] Larivi re M, Goffaux P, Marchand S, Julien N. Changes in pain perception and descending inhibitory controls start at middle age in healthy adults. Clin J Pain 2007;23:506-10.

[26] Lascelles BDX, Gaynor JS, Smith ES, Roe SC, Marcellin-Little DJ, Davidson G, Boland E, Carr J. Amantadine in a multimodal analgesic regimen for alleviation of refractory osteoarthritis pain in dogs. J Vet Intern Med 2008;22:53-9.

[27] Lervik A, Haga HA, Ranheim B, Spadavecchia C. The influence of a continuous rate infusion of dexmedetomidine on the nociceptive withdrawal reflex and temporal summation during isoflurane anaesthesia in dogs. Vet Anaes Analg 2012;39:414-25.

[28] Lewis GN, Rice DA, McNair PJ. Conditioned pain modulation in populations with chronic pain: a systematic review and meta-analysis. J Pain 2012;13:936-44.

[29] Neogi T. The epidemiology and impact of pain in osteoarthritis. Osteoarthr Cartil 2013;21:1145-53.

[30] Nir R-R, Yarnitsky D, Honigman L, Granot M. Cognitive manipulation targeted at decreasing the conditioning pain perception reduces the efficacy of conditioned pain modulation. PAIN 2012;153:170-6.

[31] Percie du Sert N, Rice ASC. Improving the translation of analgesic drugs to the clinic: animal models of neuropathic pain. Br J Pharmacol 2014; 171:2951-63.

[32] Popescu A, LeResche L, Truelove EL, Drangsholt MT. Gender differences in pain modulation by diffuse noxious inhibitory controls: a systematic review. PAIN 2010;150:309-18.

[33] Pud D, Granovsky Y, Yarnitsky D. The methodology of experimentally induced diffuse noxious inhibitory control (DNIC)-like effect in humans. PAIN 2009;144:16-19.

[34] Rasbash J, Browne WJ, Healy M, Cameron B. MLwiN Version 3.00: Centre for Multilevel Modelling, University of Bristol, United Kingdom, 2017.

[35] Rhudy JL, France CR. Defining the nociceptive flexion reflex (NFR) threshold in human participants: a comparison of different scoring criteria. PAIN 2007;128:244-53.

[36] Roeckel LA, Le Coz GM, Gavériaux-Ruff C, Simonin F. Opioid-induced hyperalgesia: cellular and molecular mechanisms. Neuroscience 2016; 338:160-82

[37] Sandrini G, Serrao M, Rossi P, Romaniello A, Cruccu G, Willer JC. The lower limb flexion reflex in humans. Prog Neurobiol 2005;77:353-95.

[38] Vainio O. Translational animal models using veterinary patients-an example of canine osteoarthritis (OA). Scand J Pain 2012;3:84-9.

[39] Winter L, Nadeson R, Tucker AP. Antinociceptive properties of neurosteroids: a comparison of alphadolone and alphaxalone in potentiation of opioid antinociception. Anesth Analg 2003;97: 798-805.

[40] Wylde V, Palmer S, Learmonth ID, Dieppe P. Somatosensory abnormalities in knee OA. Rheumatology (Oxford) 2012;51:535-43.

[41] Yarnitsky D, Crispel Y, Eisenberg E, Granovsky Y, Ben-Nun A, Sprecher E, Best L-A, Granot M. Prediction of chronic post-operative pain: pre-operative DNIC testing identifies patients at risk. PAIN 2008; 138:22-8 